\title{
Numerical study of coexisting high and low amplitude responses and safe basin erosion for a coupled linear oscillator and nonlinear absorber system
}

\author{
R. P. Eason ${ }^{\mathrm{a}}$, A. J. Dick ${ }^{\mathrm{a}, *}$, S. Nagarajaiah ${ }^{\mathrm{a}, \mathrm{b}}$ \\ ${ }^{a}$ Dept. of Mechanical Engineering and Materials Science, Rice University, Houston, \\ Texas 77005 \\ ${ }^{b}$ Dept. of Civil and Environmental Engineering, Rice University, Houston, Texas 77005
}

\begin{abstract}
Over the last half century, numerous nonlinear variants of the tuned mass damper have been developed in order to improve attenuation characteristics. In the present study, the performance of a linear oscillator and an absorber with a strongly nonlinear cubic stiffness is evaluated by using numerical methods. This configuration has been of recent interest due to its capability of wide-band energy absorption. However, high amplitude solutions, which would amplify the response of the system, have been shown to often coexist with the low amplitude solutions. The present research is focused on numerically determining the relative strength of the coexisting solutions. Erosion profiles are presented, quantifying the integrity of the system, i.e. the likelihood of converging to a safe, low amplitude response, and providing an indication of the structural safety of a practical absorber system. The results indicate that the high amplitude solutions not only exist but significantly influence the response of the system within the range of expected operating conditions, particularly at excitation frequencies lower than the natural frequency of the linear oscillator. The erosion profiles indicate a 20-40\% increase in system integrity for the case of zero damping compared to a small amount of damping, no significant integrity change when adding a small linear stiffness component to the nonlinear absorber, and no significant change in integrity between the midpoint and extreme of the bi-stable
\end{abstract}

*Please address all correspondence to this author.

Email address: andrew.j.dick@rice.edu (A. J. Dick) 
range. Additional higher-period solutions are also discovered and evidence of a chaotic response is presented.

Keywords: Nonlinear Energy Sink (NES), Multi-DOF Cell Mapping (MDCM), Tuned Mass Damper (TMD)

\section{Introduction}

Nonlinear absorbers have been shown to possess some unique benefits when used as passive vibration absorbers. Under certain conditions, however, nonlinear absorbers are capable of exhibiting multiple stable responses, some of which can be harmful for the primary oscillator. The existence and magnitude of the various solutions in these bi-stable regions have been previously identified, but the basins which indicate the relative strength of each attractor have not yet been studied. In the present paper, the basin erosion profiles of a linear oscillator coupled with an absorber having a nonlinear cubic stiffness are constructed. In this section, a review of related literature is first presented. Then, key methodology related to the construction and interpretation of basins and their corresponding erosion profiles is discussed.

\subsection{Literature Review}

A recurring challenge in the design of structures and mechanical systems is the effective attenuation of vibrations resulting from internal imbalance or external excitation. When it is not possible to remove the source of excitation or adjust system parameter values to be less susceptible to vibration, an auxiliary absorber may be beneficial. One of the most common auxiliary absorbers is the tuned mass damper (TMD), patented by Frahm in 1911 [1]. Analytical relations describing the motion of the TMD were published soon after by Ormondroyd and Den Hartog, and are still widely used today [2, 3]. The performance benefits of the TMD have been demonstrated for random excitation of single degree-of-freedom (DOF) systems [4] and multiple-DOF systems [5]. Many parametric studies have been conducted, for example optimizing the damping ratio for narrow-band attenuation [6] and determining favorable system parameters for wide-band [7] and random excitation [8]. The behavior and capabilities of TMDs are now well understood and as a result these devices are now widely implemented in a variety of structural and mechanical systems. 
Over the years, many attempts have been made to improve upon the performance of the TMD by introducing various nonlinear elements. Of particular interest to the present work are those absorbers which incorporate a nonlinear stiffness element, hereafter simply referred to as nonlinear TMDs (NTMDs). An NTMD with a cubic stiffness force profile was first proposed by Roberson as a way to increase the effective bandwidth over that of a TMD [9]. Pipes later derived analytical solutions for an NTMD with a hyperbolic sine force profile which reduced the gradient of the resonant peaks in the frequency domain [10]. However, arguably the most significant potential of the NTMD was not realized until more recently when the "energy pumping" phenomenon was discovered.

Energy pumping refers to the rapid and irreversible transfer of energy between two oscillators, the theory of which is rooted in Nayfeh and others' work regarding energy transfer between nonlinear normal modes [11, 12]. Gendelman utilized the energy pumping phenomenon to develop a nonlinear absorber capable of irreversibly extracting energy from a primary structure [13]. Along with his colleagues, Gendelman expanded on this initial work by presenting a thorough explanation of the dynamics governing the energy transfer later the same year $[14,15,16]$.

Although the NTMD has been demonstrated to exhibit unique benefits as a vibration absorber, it is now well known that in some cases dangerous high amplitude solutions exist in addition to the low amplitude "safe" solutions $[17,18]$. In a related paper by the authors, the results of a numerical study for the use of a series STMD to attenuate the response of a linear oscillator and a TMD which has been detuned due to a weak hardening type nonlinearity were presented [19]. An alternate method of eliminating the high amplitude solutions by using a piecewise-quadratic viscous damper was investigated by Starosvetsky and Gendelman [20].

In contrast to the previous literature which has identified the existence of various coexisting solutions in the NTMD system, the focus of the present paper is to identify the basins of attraction which indicate the strength of each solution. Basin portraits are constructed and various integrity measures are used to construct the erosion profiles, which concisely illustrate the degradation of the safe basin versus excitation magnitude.

\subsection{Background}

The thorough study of the erosion profiles in the present work is computationally expensive, and results can only be efficiently obtained and concisely 
presented by utilizing some of the key concepts presented in this section. The parallelized multi-degrees-of-freedom cell mapping method, a technique for efficiently calculating the basins of attraction of higher-dimensional systems, is first discussed. Then, four scalar integrity measures used to condense the extensive data into a small number of erosion profiles are introduced.

\subsubsection{Parallelized Multi-DOF Cell Mapping}

A well-known characteristic of nonlinear dynamic systems is that they are capable of possessing multiple coexisting solutions, thus creating bi-stable regions. For this reason nonlinear systems are often avoided for practical applications. However, if certain performance benefits can outweigh the burden of a complex response, it is important to characterize any existing bi-stable regions in order to most accurately predict the system behavior.

Characterizing the behavior within the bi-stable regions consists of two steps: identifying the amplitude of each stable attractor and determining the size and distribution of each existing basin, the set of initial conditions $\mathbf{q}_{0}$ converging to each attractor. The former can be accomplished by using numerical continuation techniques or approximate analytical methods. In the present case, AUTO bifurcation and continuation software, which employs a psuedo-arclength continuation method, is used [21]. The latter is accomplished by constructing basin portraits, two dimensional cross-sections of the $n$-dimensional basins of attraction. The basin portrait planes are denoted by $\Sigma^{a b}$, the superscripts identifying the two dimensions $a$ and $b$ in which the planes lie.

The most straightforward method used to construct basin portraits is the grid of starts (GOS) [22]. In this method, a grid of initial conditions (IC) within the portrait plane is defined and from each initial point a solution is obtained by using numerical integration. The steady-state behavior of each solution identifies to which attractor the initial point converges and so the basins of attraction are constructed accordingly. While this method is easily implemented and can be accurate when given sufficient time for the transient behavior to decay; the computational cost is high, particularly for higher-dimensional systems and parametric studies. For these conditions, a cell mapping approach is more appropriate.

A variety of cell mapping methods have been developed over the years in order to study the global behavior of nonlinear dynamic systems. For cell mapping methods, the state space is discretized into a cell-state space and all possible trajectories are simultaneously approximated by defining the 
mapping between cells by using numerical integrations over a small time step. A specific particle's trajectory through continuous state space can then be constructed by following the map through cell-state space.

Two of the first and most widely used cell mapping methods were Hsu's simple cell mapping (SCM) [23, 24] and generalized cell mapping (GCM) [25], the latter being a more complex method which provides a better analysis of chaotic systems or those with fractal basin boundaries. Other cell mapping methods include interpolated, modified interpolated, and higher-order interpolated cell mapping $[22,26,27,28]$, and parameter variation methods PVSCM and PVICM [29, 30]. Although the benefits of each of these methods are easily realized for single-DOF systems, their efficiency is greatly diminished for higher-dimensional systems due to an exponential scaling of the total number of cells required.

The multi-degrees-of-freedom cell mapping method (MDCM) was developed by van der Spek and colleagues in order to overcome this scaling limitation, preserving the efficiency of cell mapping methods for systems with an arbitrary number of dimensions [30,31]. Similar to the other cell mapping methods, basin portraits are constructed by mapping short-time trajectories starting from the center of cells in cell-state space. However, in the MDCM method there is no need to initiate trajectories from every cell within the $n$-dimensional cell-state space, rather only those in a two-dimensional subset of cells, $S$, corresponding to the basin portrait to be produced. In order to account for trajectories that map outside $S$, the MDCM method uses a unique, unbounded numbering convention in which $n$ integer coordinates are assigned to label each cell.

Recently the authors have developed a modified version of the MDCM method, the so-called parallelized multi-degrees-of-freedom cell mapping method (PMDCM) [32]. The PMDCM method uses the same theory and basic operations as MDCM to construct basin portraits but employs a restructured algorithm which allows for the use of parallel computing resources. As a result, the computational efficiency for higher-dimensional systems is greatly improved and the fewer sequential operations reduce the accumulation of error and provides more accurate results. For this reason, the PMDCM method is used to construct all basin portraits in this study, and the accuracy of key results are verified using results obtained from the GOS method.

Methods have also been developed to experimentally obtain basin portrait information through the application of Poincaré sections to transient response data [33]. In the corresponding numerical effort, the addition of 
noise was demonstrated to disrupt clear boundaries between basins and result in fractal-like mixing at the boundaries.

\subsubsection{Integrity Measures}

Basin portraits provide a useful illustration of the strength of various attractors, representing the dynamical integrity of a system. At least two distinct regions are illustrated in each portrait: the safe basin and the constraint basin. The safe basin, denoted by $\mathscr{A}_{\infty}$, represents the set of all initial conditions that converge to a "safe solution" possessing favorable response characteristics. Generally, the safe solution is defined by a steady-state response amplitude below a threshold value. The constraint basin, $\mathscr{C}_{\infty}$, represents the set of initial conditions that converge to a solution above the threshold value. In some cases, multiple safe or constraint basins are illustrated in a single portrait in order to distinguish between regions converging to specific attractors. Basin portraits have been used to study dynamical integrity in nonlinear systems ranging from the macro-scale down to the nanoscale. These systems include unsynchronized power systems, planar pendula, vibration-based energy harvesters, nonlinear mechanical oscillators, atomic force microscopes, and actuated carbon nanotubes [34, 35, 36, 37, 38, 39].

When the results from a large number of basin portraits are to be summarized, it becomes necessary to concisely identify key characteristics of the portraits by using scalar integrity measures. Four key scalar integrity measures are the global integrity measure (GIM), local integrity measure (LIM) and impulsive integrity measure (IIM) developed by Soliman and Thompson in 1989 [40], and the integrity factor (IF), introduced by Lenci and Rega in the last decade [41]. In general, GIM $\geq$ IF $\geq$ IIM $\geq$ LIM. Figure 1 illustrates three of the integrity measures superimposed on the basin portrait of a hypothetical two-DOF system. The basin portrait consists of a disjoint safe basin (white) and a constraint basin (shaded). The safe attractor is denoted with a star.

The global integrity measure and the local integrity measure are two of the most commonly used integrity measures in this type of global analysis [40]. The GIM is defined as the normalized hyper-volume of the safe basin $\mathscr{A}_{\infty}$. In a two-dimensional system, the GIM therefore represents the area of $\mathscr{A}_{\infty}$ normalized by a reference area (e.g. the area within the circle of radius $R$ ). The LIM is a much more conservative measure, defined as the normalized radius of the largest hyper-sphere (circle in two dimensions) that is centered on the safe attractor and entirely contained within the safe basin. A direct 


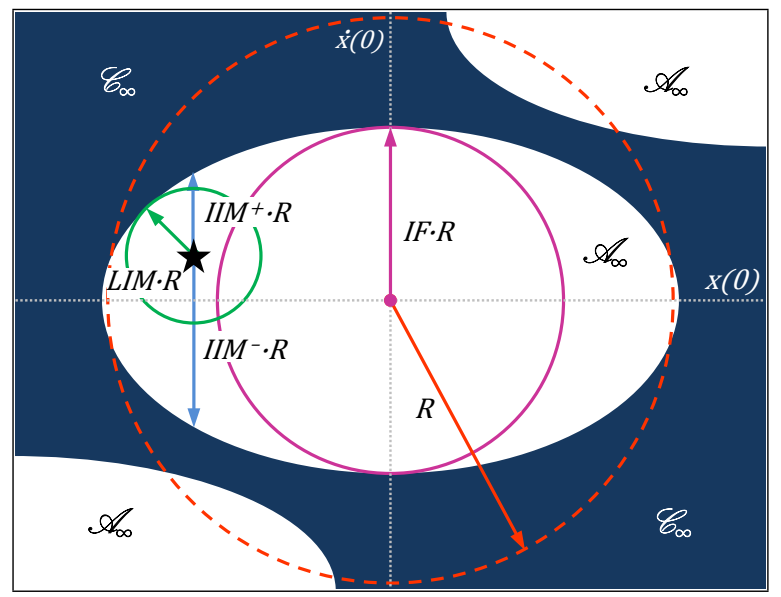

Figure 1: Three integrity measures illustrated on the basin portrait of a hypothetical two degrees-of-freedom system.

correlation therefore exists between the LIM and the stability of the safe attractor with regard to its robustness to perturbations.

Also directly correlated to the stability of the safe attractor is the impulsive integrity measure. Recalling that an ideal impulse is defined as an instantaneous change in velocity, the IIM is defined as the normalized distance from the safe attractor to the boundary of $\mathscr{C}_{\infty}$ in the direction of a generalized coordinate corresponding to a velocity. The IIM corresponding to an impulse in the positive direction is denoted by $\mathrm{IIM}^{+}$, and in the negative direction by $\mathrm{IIM}^{-}$. The measure $\mathrm{IIM}^{ \pm}$is used to indicate the minimum of the two impulse integrity measures.

The integrity factor was introduced as an alternative to the GIM in order to account only for the compact part of the safe basin. The IF is defined as the normalized radius of the largest hyper-sphere (circle in two dimensions) that can be completely contained within the safe basin and therefore provides a more conservative integrity estimation than the GIM when dealing with disjoint basins, or fractal or entangled boundaries.

\section{Modeling}

The two degrees-of-freedom dynamic system consists of a linear primary oscillator of mass $M$, hereafter referred to as the Primary, coupled with an NTMD of mass $m_{N}$ and is illustrated in Fig. 2. This configuration represents 


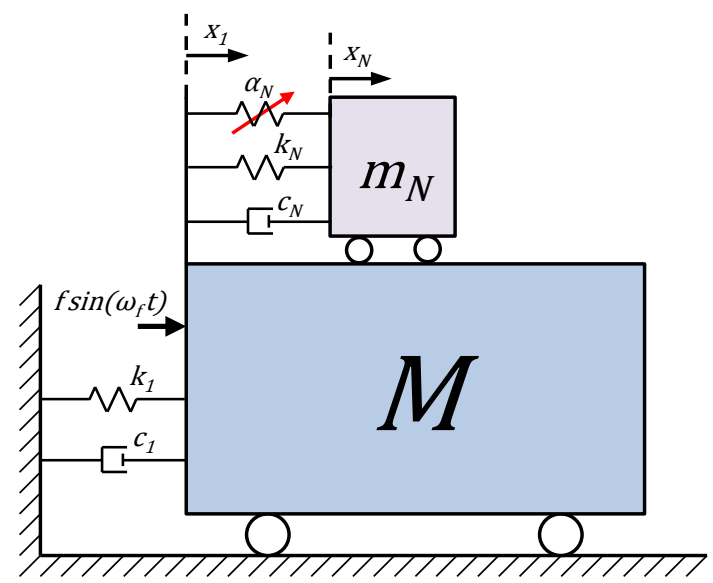

Figure 2: Schematic of a primary oscillator (Primary) and nonlinear tuned mass damper (NTMD).

a simplified model of a general structure and nonlinear absorber, allowing for the effects of the nonlinear interaction to be isolated without the influence of any extraneous behavior specific to a particular implementation. The results are relevant for those structural or mechanical systems in which the response is dominated by the first mode.

Damping coefficients of the primary oscillator and NTMD are represented by $c_{1}$ and $c_{N}$, respectively. The primary oscillator is grounded with a linear stiffness coefficient $k_{1}$. The NTMD is coupled to the primary oscillator such that the restoring force $f_{N}$ consists of a linear and a cubic component, as described by

$$
f_{N}=k_{N}\left(x_{1}-x_{N}\right)+\alpha_{N}\left(x_{1}-x_{N}\right)^{3} .
$$

Deriving the equations of motion is straightforward by using the standard Newtonian or Lagrangian procedure. The second order equations of motion 
describing the Primary-NTMD system are presented in Eqs. (2)-(3).

$$
\begin{aligned}
\ddot{x}_{1}= & -\frac{c_{1}}{M} \dot{x}_{1}-\frac{c_{N}}{M}\left(\dot{x}_{1}-\dot{x}_{N}\right)-\frac{k_{1}}{M} x_{1}-\frac{k_{N}}{M}\left(x_{1}-x_{N}\right) \\
& -\frac{\alpha_{N}}{M}\left(x_{1}-x_{N}\right)^{3}+\frac{f}{M} \sin \left(\omega_{f} t\right), \\
\ddot{x}_{N}= & -\frac{c_{N}}{m_{N}}\left(\dot{x}_{N}-\dot{x}_{1}\right)-\frac{k_{N}}{m_{N}}\left(x_{N}-x_{1}\right) \\
& -\frac{\alpha_{N}}{m_{N}}\left(x_{N}-x_{1}\right)^{3} .
\end{aligned}
$$

In order to present the results in a general manner that can be easily interpreted for a variety of implementations, the equations of motion are nondimensionalized. The relations given in Eqs. (4)-(7) are used to nondimensionalize the coefficients with respect to time.

$$
\begin{gathered}
\omega_{1}=\sqrt{\frac{k_{1}}{M}}, \quad \omega_{N}=\sqrt{\frac{\alpha_{N}}{m_{N}}}, \quad \omega_{2}=\sqrt{\frac{k_{N}}{m_{N}}}, \\
\omega=\frac{\omega_{f}}{\omega_{1}}, \quad \Omega=\frac{\omega_{2}}{\omega_{1}}, \quad \Omega_{N}=\frac{\omega_{N}}{\omega_{1}}, \\
\gamma_{1}=\frac{c_{1}}{2 \omega_{1} M}, \quad \gamma_{N}=\frac{c_{N}}{2 \omega_{2} m_{N}}, \quad \hat{\gamma}_{N}=\gamma_{N} \Omega=\frac{c_{N}}{2 \omega_{1} m_{N}}, \\
\epsilon=\frac{m_{N}}{M}, \quad \tau=\omega_{1} t, \quad F=\frac{f}{k_{1}} .
\end{gathered}
$$

In order to conclude the nondimensionalization, the displacement variables $x_{i}$ are normalized by the static displacement, $F$, resulting in the generalized coordinates $q_{i}=x_{i} / F$. Substituting the generalized coordinates and Eqs. (4)-(7) into Eqs. (2)-(3) results in the fully nondimensionalized form of the equations of motion. These final equations are expressed as a system of first order differential equations in Eqs. (8)-(11), with $q_{1} \equiv q_{P}$ and $q_{3} \equiv q_{N}$.

$$
\begin{aligned}
\dot{q}_{1}= & q_{2}, \\
\dot{q}_{2}= & -2 \gamma_{1} q_{2}-2 \epsilon \hat{\gamma}_{N}\left(q_{2}-q_{4}\right)-q_{1} \\
& -\epsilon \Omega^{2}\left(q_{1}-q_{3}\right)-F^{2} \epsilon \Omega_{N}^{2}\left(q_{1}-q_{3}\right)^{3} \\
& +\sin (\omega \tau), \\
\dot{q}_{3}= & q_{4}, \\
\dot{q}_{4}= & -2 \hat{\gamma}_{N}\left(q_{4}-q_{2}\right)-\Omega^{2}\left(q_{3}-q_{1}\right) \\
& -F^{2} \Omega_{N}^{2}\left(q_{3}-q_{1}\right)^{3} .
\end{aligned}
$$


Note that the NTMD damping parameter $\hat{\gamma}_{N}$ is used instead of the linear damping ratio of the NTMD $\gamma_{N}$. Since $\omega_{1}$ and $m_{N}$ remain constant throughout the study, $\hat{\gamma}_{N}$ allows for the use of a constant, nondimensionalized damping coefficient even when the value of the linear NTMD stiffness is set to zero.

Numerical integration and continuation techniques are used to evaluate the equations of motion, as described in the following sections.

\section{Results}

In this section, the global dynamic behavior of the system is studied by using numerical methods. First, the results from a preliminary investigation used to determine the most useful parameter values are presented. Then, the global dynamics of the system are discussed and the erosion profiles are presented.

\subsection{System and Environmental Parameter Values}

The behavior of the present system is a function of the five system parameters $\left\{\epsilon, \gamma_{1}, \hat{\gamma}_{N}, \Omega, \Omega_{N}\right\}$ and two environmental parameters $\{F, \omega\}$. The first challenge is therefore to determine a small number of design and environmental parameter value combinations for which to construct a meaningful set of erosion profiles. In order to accomplish this, realistic limits for each of the parameters are given and then a preliminary numerical study is used in order to identify the target parameter combinations.

Following the example of related work [17], a mass ratio value of $\epsilon=0.1$ is used, representing an approximate upper limit of the largest absorber that would be installed in a structure. Common approximations of structural damping ratios are either $2 \%$ or $5 \%$, so a primary oscillator damping ratio of $\gamma_{1}=0.02$ is selected [5]. Similarly, NTMD damping ratio values of $\gamma_{N} \in$ $\{0,0.02,0.05\}$ are used, including the case of zero-damping as a lower limit and noting that small damping values such as these are commonly targeted to maximize energy transfer to the absorber. The value of the NTMD damping coefficient $\hat{\gamma}_{N}$ are selected based on the results of this preliminary study.

The linear stiffness parameter is varied from $\Omega \in[0,1]$, with the lower limit representing an essentially nonlinear absorber and the upper limit bounded by the natural frequency of the primary structure. Following again the nonlinear absorber design process used to achieve energy pumping in [17], the nonlinear stiffness parameter is expected to be on the order of $\Omega_{N} \approx 0.1$, 
so the range of values $\Omega_{N} \in[0,0.5]$ is selected, although an upper limit of $\Omega_{N}=2$ was initially investigated in order to not overlook any favorable response behavior at higher levels of nonlinearity.

Three representative values for the static displacement are used, $F \in$ $\{0.35,0.6,2.5\}$. The first, $F=0.35$, represents a structural system exposed to a seismic event that is approximately half of the expected strength. Most importantly, this value matches that used to produce some of the key results in [17] within which the detached resonance is illustrated. The second, $F=$ 0.6 , is used to denote the most-likely case. The largest magnitude value, $F=$ 2.5 , represents the worst case scenario where the dominant frequency of the seismic event corresponds to the natural frequency of the primary structure. Each of these scalar values was calculated by calculating the root-meansquared value of the frequency-dependent static displacement amplitude over $\omega \in[0,2]$ for the Kanai-Tajimi event described in [17].

In order for the erosion profiles constructed in the following subsection to be most useful, sets of parameter values must be located that produce a response which meets the following two criteria:

1. Multiple stable solutions coexist (bi-stable regions) within the range of expected operating conditions as defined by the environmental parameters.

2. The NTMD outperforms an optimally tuned linear TMD based on one or more performance measures when considering only the lowestamplitude solution branch in each bi-stable region.

The first condition is a necessary criterion in order to be able to construct the erosion profiles, which can only be done when more than one stable solution is present. The second condition ensures that the erosion profiles will be useful, since the end goal of the present and related research is to determine whether an NTMD can be developed which outperforms an optimally tuned linear TMD. In this context, if an NTMD does not satisfy the second condition then it provides no practical value and therefore its corresponding erosion profile is of no interest.

Based on recent literature, a number of NTMD performance benefits over a standard linear TMD have been cited. For certain parameter values, it is possible to achieve a reduction in the peak amplitude and the RMS of the frequency-response as well as achieve an increase in the suppression bandwidth. Initial simulations indicated unreliability in the use of an automated 

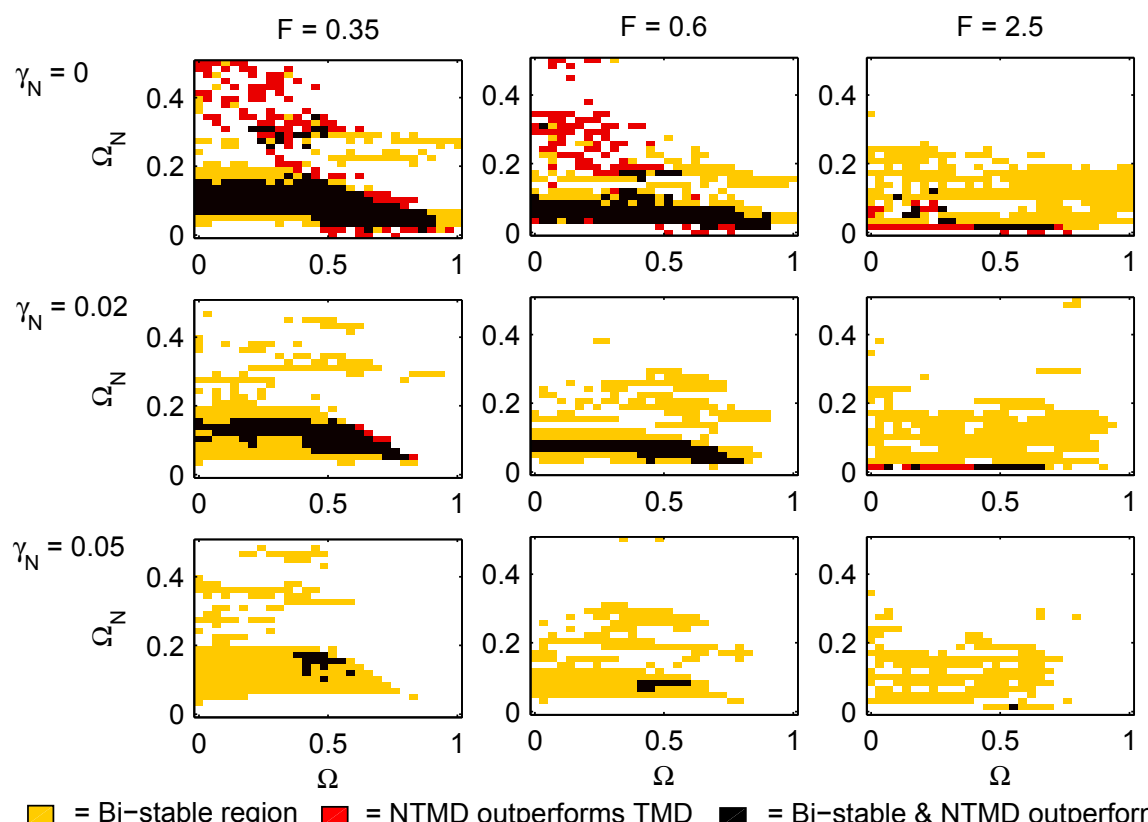

$=\mathrm{Bi}$-stable region

$\square=$ NTMD outperforms TMD

$=\mathrm{Bi}$-stable $\&$ NTMD outperforms TMD

Figure 3: Estimated regions of parameter space that meet the criteria noted in the legend from an initial study.

algorithm to calculate the suppression bandwidth. For this reason, the peak amplitude and RMS of the frequency-response are selected as the two measures used to compare the performance of the NTMD and TMD.

A numerical algorithm is developed to scan the parameter space in order to identify approximate regions that meet these two criteria. For each of the nine combinations of $\gamma_{N}$ and $F$, a grid of $30 \times 30 \Omega$ by $\Omega_{N}$ values over the domain of each parameter is constructed. At each point $\left[\Omega, \Omega_{N}\right]$ within each grid, numerical integration is used to construct the solution at each of 50 excitation frequencies $\omega \in[0.5,1.5]$ and 30 initial conditions $q_{1}^{0} \in[0,35], q_{i}^{0}=$ 0 for $i=2,3,4$. This range and resolution of initial conditions is determined to be sufficient in order to identify the presence of multiple solutions by examining a single dimension cross-section of the basin. This is later verified by examining the basins constructed for the work in the final part of this section. The collection of amplitudes $\left|q_{1}\right|$ calculated from each solution at the combinations of $\omega$ and $q_{1}(0)$ are used to determine which, if any, of the above criteria are met by the point $\left[\Omega, \Omega_{N}\right]$. 
The results of the parameter space scan are illustrated in Fig. 3. Each of the subplots represents a combination of $\gamma_{N}$ and $F$, as indicated by the respective row and column labels. Light (yellow) shading indicates that only the first criterion is satisfied at the given point, medium (red) shading indicates that only the second criterion is satisfied, and dark (black) shading indicates that both criteria are satisfied, providing the "region of interest". The absence of shading indicates that neither criterion is satisfied.

Two general trends are observed from the results in Fig. 3. First, the area of the region of interest decreases as the static displacement increases, and the region shifts toward lower values of $\Omega_{N}$. This indicates that larger static displacement values require a smaller nonlinear stiffness to maintain performance and the resulting regions of interest are less robust to detuning of the linear or nonlinear stiffness. Second, larger NTMD damping ratio values correlate with a smaller region of interest and a shift toward larger values of $\Omega$. This suggests that the damping in an NTMD should be kept as small as possible to produce more conditions where the NTMD may outperform a comparable TMD.

The regions of interest estimated in Fig. 3 are refined by examining frequency-response plots constructed by using numerical continuation techniques at a number of points in each grid. In general, the frequency-response profiles within the regions of interest display common characteristics, similar to those illustrated in Fig. 4. The frequency-response curves presented in this and subsequent figures are obtained by using a continuation method which results in a complete bifurcation diagram for the system and excitation conditions considered. While this does not correspond to the broadband excitation frequently experienced in practical application, these figures provide a complete picture of the system's steady-state response behavior. The Period-1 solutions depicted in Fig. 4 were observed by Alexander and Schilder, who studied the profile changes that occurred for various parameter values [17]. In this most general case, two bi-stable regions exist.

The higher-frequency bi-stable region, denoted $B_{\omega>1}$, is observed for $\omega \in$ $[1.4,2.6]$ in the present example. In general, for larger values of $\Omega_{N}$ the magnitude of the high amplitude solution branch decreases and the limit point bifurcation at the upper bound of the region shifts right, increasing the width of the region. For the cases with non-zero NTMD damping, smaller values of $\Omega$ also correspond to a rightward shift of the limit point bifurcation. Based on a preliminary study, the integrity of the low amplitude solution within $B_{\omega>1}$ is significantly higher than in the lower-frequency region, $B_{\omega<1}$, and 

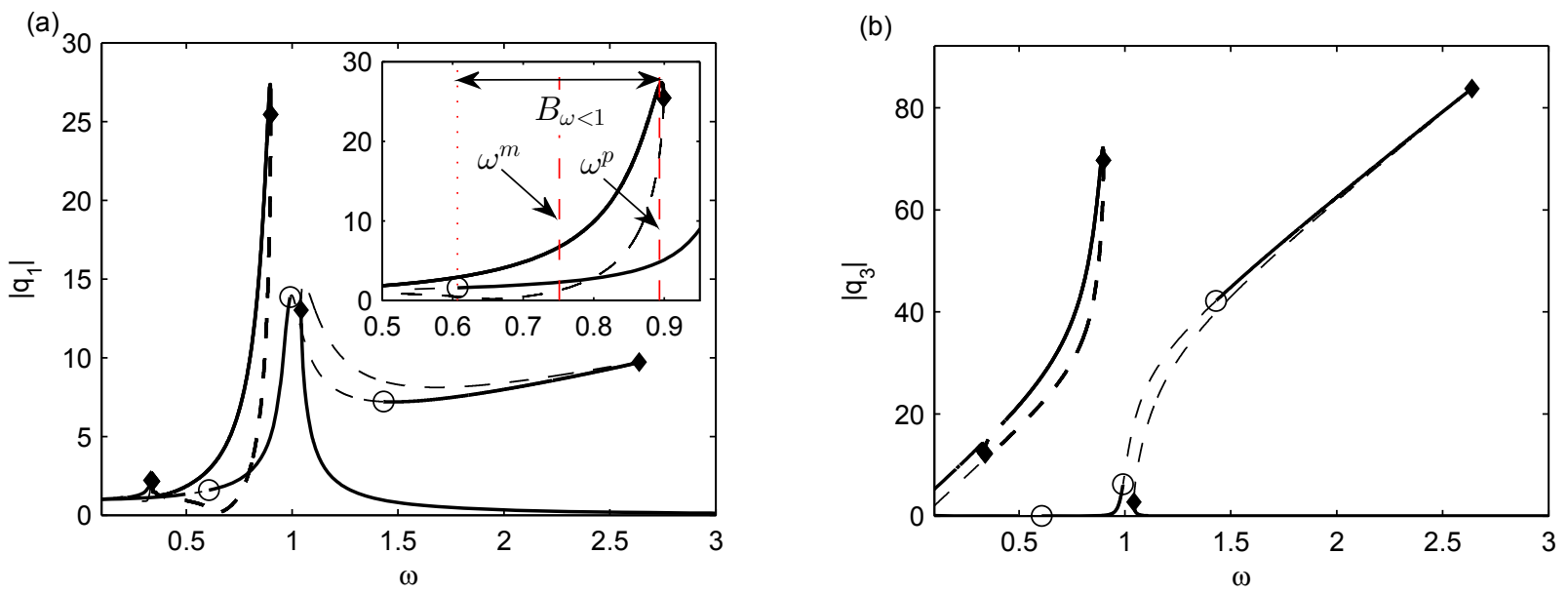

Figure 4: Characteristic frequency-response profile for the (a) Primary and (b) NTMD illustrating the two bi-stable regions; $\Omega=0, \hat{\gamma}_{N}=0.002, \Omega_{N}=0.09, F=0.35, \phi=0$. Limit point $(\diamond)$ and torus $(\bigcirc)$ bifurcation points are marked.

Table 1: Four sets of parameter values for which the erosion profiles are constructed.

\begin{tabular}{ccccc}
\hline$\epsilon$ & $\gamma_{1}$ & $\Omega$ & $\hat{\gamma}_{N}$ & $\Omega_{N}$ \\
\hline 0.1 & 0.02 & 0 & 0 & 0.06 \\
0.1 & 0.02 & 0 & 0.002 & 0.09 \\
0.1 & 0.02 & 0.1 & 0 & 0.06 \\
0.1 & 0.02 & 0.1 & 0.002 & 0.09 \\
\hline
\end{tabular}

therefore the main focus of this paper is on the integrity of solutions in $B_{\omega<1}$. A brief overview of the behavior within $B_{\omega>1}$ is presented in Section 3.2.

The lower-frequency bi-stable region $B_{\omega<1}$ is observed for $\omega \in[0.6,0.9]$ in the present example. Initial results revealed that either the lower-amplitude (safe solution) or higher-amplitude (constraint solution) branch could dominate the system response depending on the system parameters. Therefore, the present study is focused on determining the erosion profiles within $B_{\omega<1}$. These results are presented in Section 3.3.

Results are obtained at two key excitation frequencies within $B_{\omega<1}$, denoted $\omega_{m}$ and $\omega_{p}$ and illustrated in Fig. 4(a). The first, $\omega_{m}$, corresponds to the midpoint of the bi-stable region bounded by the torus bifurcation (also 
known as a secondary Hopf bifurcation) of the safe solution at the lower limit and by the limit point bifurcation of the constraint solution at the upper limit. The second, $\omega_{p}$, is the frequency corresponding to the peak amplitude of the constraint solution.

The four sets of system parameter values from which the erosion profiles are constructed are listed in Table 1 . In order to examine both an ideal case and a realistic case, the four sets are combinations of zero and nonzero values of $\hat{\gamma}_{N}$ and $\Omega$. When $\Omega=0$ the system represents an essentially nonlinear absorber, or "nonlinear energy sink". Frequency response profiles indicate that larger linear stiffness values decrease the width of the bi-stable region, so a small value, $\Omega=0.1$, is selected in order to produce a more favorable response. The NTMD damping parameter $\hat{\gamma}_{N}=0.002$ corresponds to a $2 \%$ damping ratio at $\Omega=0.1$. The value of the nonlinear parameter $\Omega_{N}$ is 0.06 for zero NTMD damping and 0.09 for $\hat{\gamma}_{N}=0.002$, corresponding to the location of the center of the region of interest depicted in Fig. 3, which shifts according to $\gamma_{N}$.

\subsection{Higher-Frequency Bi-Stable Region, $B_{\omega>1}$}

The frequency-response within $B_{\omega>1}$ is illustrated in Fig. 5 for (a) the Primary amplitude $\left|q_{1}\right|$ and (b) the NTMD amplitude $\left|q_{3}\right|$. The stable and unstable solution branches identified using numerical continuation techniques are represented by using solid and dashed lines, respectively. Amplitudes obtained by using numerical integration for $q_{1}(0) \in[-200,0], q_{i}(0)=0$ for $i=2,3,4$ are presented as black dots. In addition to the Period-1 curves illustrated in Fig. 4, a Period-3 branch and two smaller branches, Period-5 and Period-2, are observed for the system presented in Fig. 2. To the knowledge of the authors, these higher-period branches have not been identified in previous literature.

A characteristic basin portrait for $B_{\omega>1}$ in the $\Sigma^{12}$ plane is presented in Fig. 6. The excitation frequency is $\omega=1.8$, near the center of the bistable region. Basins corresponding to three stable attractors are clearly observed, corresponding to the three branches shown in Fig. 5(a). Basin $\mathscr{A}_{\infty}^{1}$ (black) corresponding to the lowest amplitude solution where the response amplitude, $\left|q_{1}\right|$, is equal to 0.4 and consists of a compact central region (radius $=15, I F \approx 0.57$ ) surrounded by a larger region where $\mathscr{A}_{\infty}^{1}$ and $\mathscr{A}_{\infty}^{2}$ (grey), corresponding to $\left|q_{1}\right|=1.6$, are intermixed. The constraint basin $\mathscr{C}_{\infty}$ (white), $\left|q_{1}\right|=7.7$ consists of a relatively small area and is not encountered until a radius of 46.8 , an $I F \gg 1$ as indicated by a comparison with the reference 

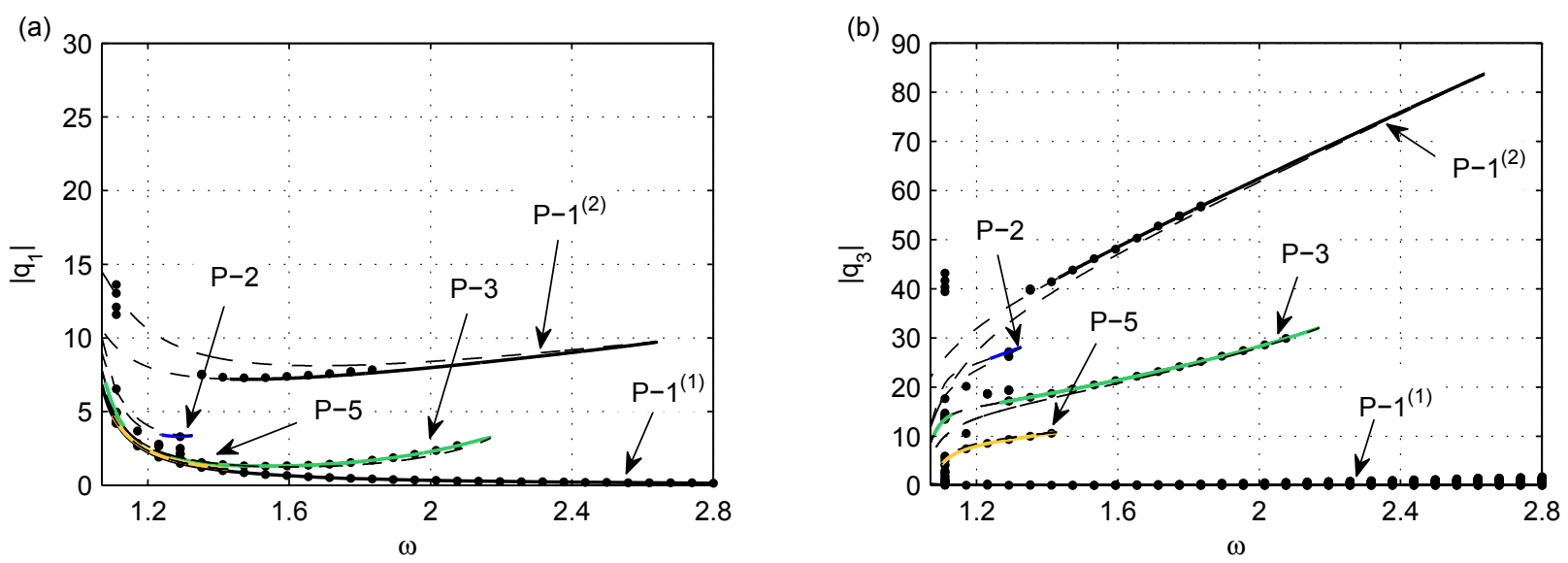

Figure 5: Frequency-response of (a) Primary and (b) NTMD within $B_{\omega>1}$ using numerical continuation (-,--) and numerical integration ( . ); $\Omega=0, \hat{\gamma}_{N}=0.002, \Omega_{N}=0.09, F=$ $0.35, \phi=0$.

circle (dashed). The dashed circle is used as a size reference and it has a radius of $R=26.3$. This value is selected in order to normalize the integrity measures for all basin portraits in this study.

The trend throughout $B_{\omega>1}$ is well represented by Fig. 6 . It takes a much larger amount of energy than in $B_{\omega<1}$ to transition the Primary into a potentially harmful high amplitude response. Even if a large impact occurred, the primary oscillator would be more likely to settle to a safe, low amplitude response due to the narrowness of the constraint basins. Similarly, if the primary oscillator were to settle to a high amplitude response, the slightest perturbation would return the system to the low amplitude state.

\subsection{Lower-Frequency Bi-Stable Region, $B_{\omega<1}$}

The frequency-response of the system within $B_{\omega<1}$ is illustrated in Fig. 7. The two Period-1 solution branches that were presented in Fig. 4 are depicted here in black. Additionally, a set of detached higher-period solution branches are superimposed. Again, the black dots represent steady-state amplitudes obtained using numerical integration over a range of initial conditions in $q_{1}(0) \in[-200,0], q_{i}(0)=0$ for $i=2,3,4$ for each $\omega$. By comparing the results obtained by using numerical integration with those obtained with the continuation method, we see that the majority of the solution branches have 


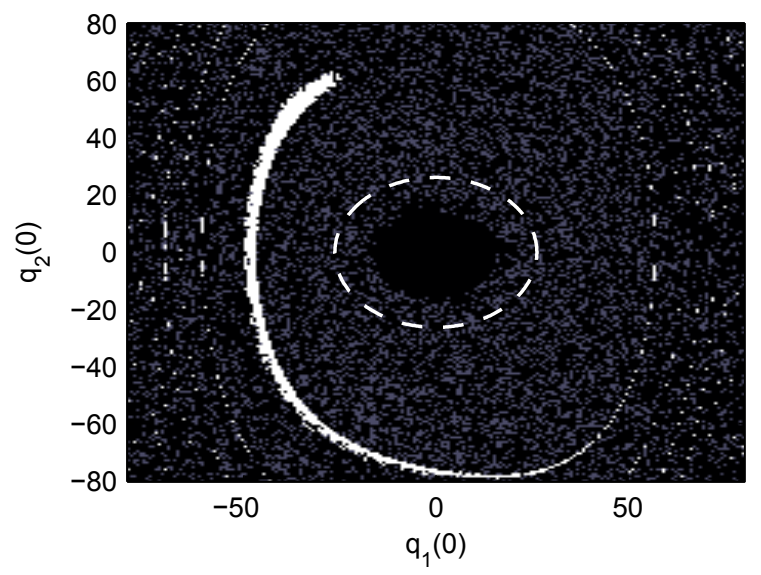

Figure 6: Characteristic $B_{\omega>1}$ basin portrait (201x201) for the $\Sigma^{12}$ plane; $\omega=1.8, \Omega=$ $0, \hat{\gamma}_{N}=0.002, \Omega_{N}=0.09, F=0.35, \phi=0$. Dashed circle is used for size reference, its radius equal to integrity measure normalization radius, $R=26.3$.

been identified here. The authors were not able to locate some branches due to their close proximity to other solutions, low stability, or other factors preventing numerical convergence in the continuation methods.

While the higher-period branches do introduce a variety of new and interesting non-zero NTMD response amplitudes in this region, all Primary amplitude values remain in the neighborhood of the Period-1 branches. Since the end goal is to the attenuate the Primary at the expense of NTMD motion, the Primary amplitude remains the focus, by using the distinct separation between the amplitude of the safe and constraint Period- 1 branches to define the safe basin $\mathscr{A}_{\infty}$ and conversely, the constraint basin $\mathscr{C}_{\infty}$. Within the algorithm, the safe basin is defined as,

$$
\mathscr{A}_{\infty}:=\left\{\left.\mathbf{q}^{0}|| q_{1}\right|_{\mathbf{q}(0)=\mathbf{q}^{0}} \geq 1.5\left|q_{1}\right|_{\mathbf{q}(0)=\mathbf{0}}\right\},
$$

where $\mathbf{q}^{0}$ represents the vector of initial conditions and $\mathbf{0}$ represents the zero vector. Both automated and manual quality checks are used to ensure that transient behavior has sufficiently decayed and that each point is added to the correct basin.

In order to calculate the erosion profiles for each of the four parameter value combinations $\left(\hat{\gamma}_{N}, \Omega\right)$, a series of two-dimensional basin portraits are constructed. Based on the results of an initial convergence study and com- 

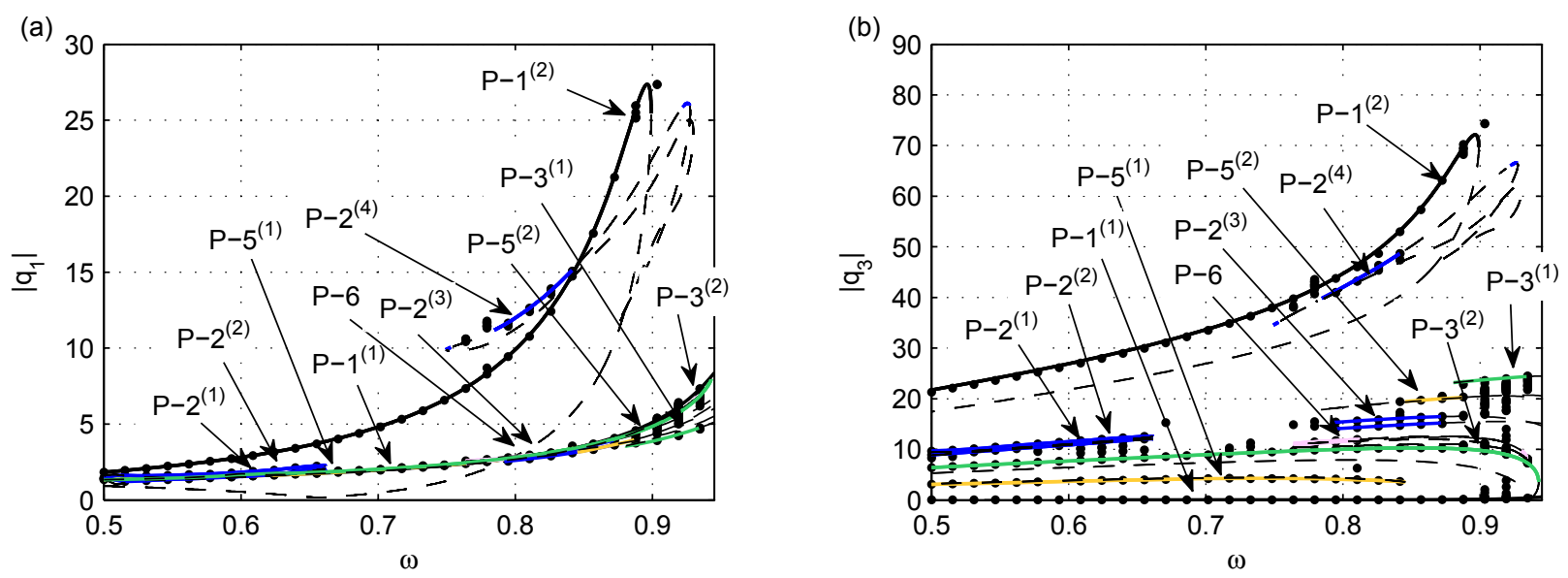

Figure 7: Frequency-response of (a) Primary and (b) NTMD $B_{\omega<1}$ using numerical continuation (-,--) and numerical integration ( . ); $\Omega=0, \hat{\gamma}_{N}=0.002, \Omega_{N}=0.09, F=$ $0.35, \phi=0$.

paring the results with the grid of starts (GOS) method, it is determined that a resolution of $101 \times 101$ is sufficient to estimate the value of each integrity measure within $\pm 1 \%$ of its true value. Limits for the $\Sigma^{12}$ and $\Sigma^{34}$ portraits are set to $q_{i}^{0} \in[-30,30], i=1,2$, and $q_{i}^{0} \in[-60,60], i=3,4$, respectively, such that the range is large enough to capture the compact part of the safe basin for the lowest forcing cases.

Since each basin portrait displays a two-dimensional cross-section of a four-dimensional hyper-space, the particular choice of the two fixed IC values, (i.e. $q_{f}^{0}, f \neq a, b$ in portrait $\left.\Sigma^{a b}\right)$ influences the portrait itself. In order to provide a general measure of integrity without the bias of defining the two fixed ICs at a specific point on a trajectory, the GIM and IF are calculated from basins with $q_{f}^{0}=0$. The LIM and IIM, on the other hand, specifically relate to the position of the attractor within the portrait and therefore can only be calculated for portraits intersected by the safe attractor. These measures are calculated in the present study by taking the minimum integrity values calculated from each of eight planes intersected by the low amplitude Period- 1 attractor, evenly spaced over the period $\mathrm{T}$ at $\tau=0, T / 8, \ldots, 7 T / 8$. All integrity measures represent the minimum value calculated from each of four phase offsets $\phi=\{0, \pi / 2, \pi, 3 \pi / 2\}$ and two portrait planes $\Sigma^{12}$ and $\Sigma^{34}$.

The characteristic basin portrait is illustrated in Fig. 8 for the (a) $\Sigma^{12}$ and 

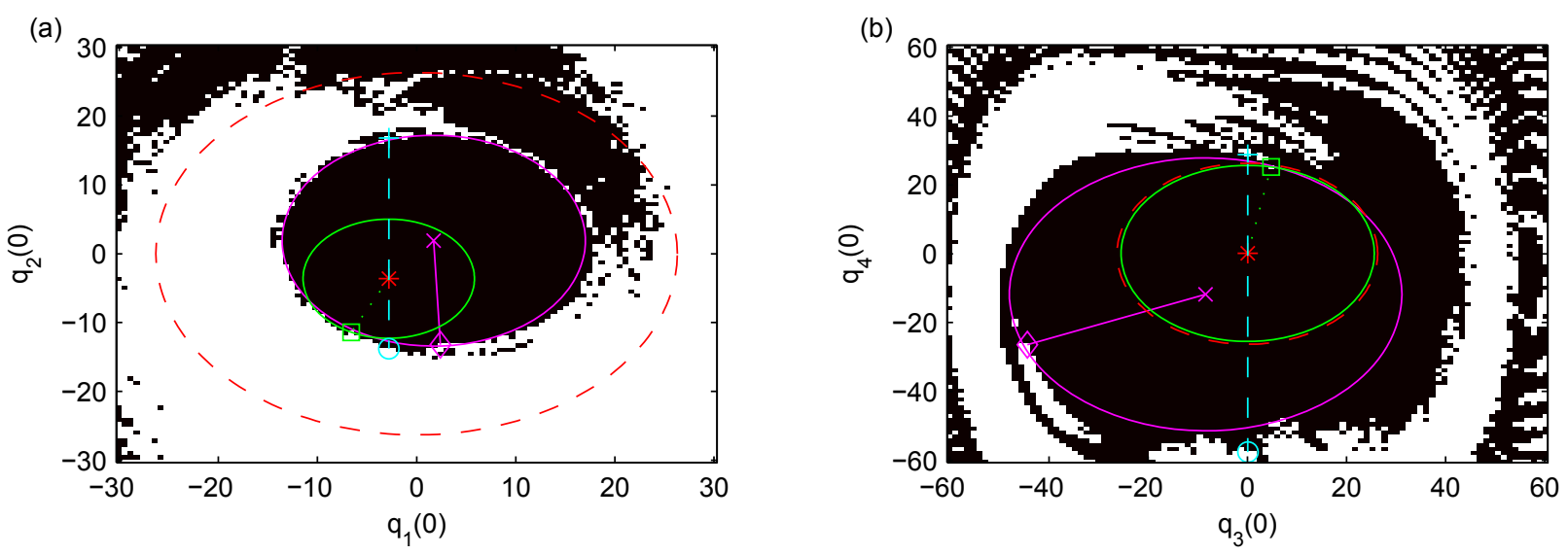

Figure 8: Characteristic basin portrait in the (a) $\Sigma^{12}$ and (b) $\Sigma^{34}$ planes illustrating the $L I M, I F$ and $I I M^{ \pm}$integrity measures. Dashed circle is used for size reference, its radius equal to integrity measure normalization radius, $R=26.3$.

(b) $\Sigma^{34}$ planes. Black and white shading denotes the safe basin $\mathscr{A}_{\infty}$ and the constraint basin $\mathscr{C}_{\infty}$, respectively. In this example, $\Omega=0, \hat{\gamma}_{N}=0.002, \Omega_{N}=$ $0.09, F=0.35$ and $\phi=0$. The basin portraits intersect the periodic attractor $(*)$ at $\mathbf{q}=[-2.81,-3.63,0.07,0.09]$. The size of the smallest circle represents the LIM, with a radius drawn from the attractor to the closest point belonging to $\mathscr{C}_{\infty}$. Vertical dashed lines drawn between the attractor and the closest point in $\mathscr{C}_{\infty}$ in the positive and negative directions illustrate $\mathrm{IIM}^{+}$and $\mathrm{IIM}^{-}$, respectively. The larger solid circle represents the IF, calculated by using the center of area of $\mathscr{A}_{\infty}$ as an initial guess and by using a numerical iterative method of enlarging and translating a circle until the space is filled. The dashed circle serves as a reference to normalize the integrity measures.

For the basin portrait illustrated in Fig. 9(a), five response time series (b) $(\mathrm{i}-\mathrm{v})$ corresponding to each of the marked initial conditions are presented in Fig. 9(b). Parameters for the basin portrait are identical to Fig. 8(a) with the exception of the phase offset, which here is $\phi=\pi / 2$ in order to obtain a more interesting collection of points on the $q_{1}(0)$ axis. The time series results verify the basin portrait constructed using the PMDCM method and help to illustrate the transient behavior.

Given the four parameter combinations $\left(\hat{\gamma}_{N}, \Omega\right)$, two frequencies $\omega_{m}$ and $\omega_{p}$, four phase offsets $\phi$, two portrait planes $\Sigma$ and nine fixed parameter combinations (including zero plus eight trajectory intersection points), a total 

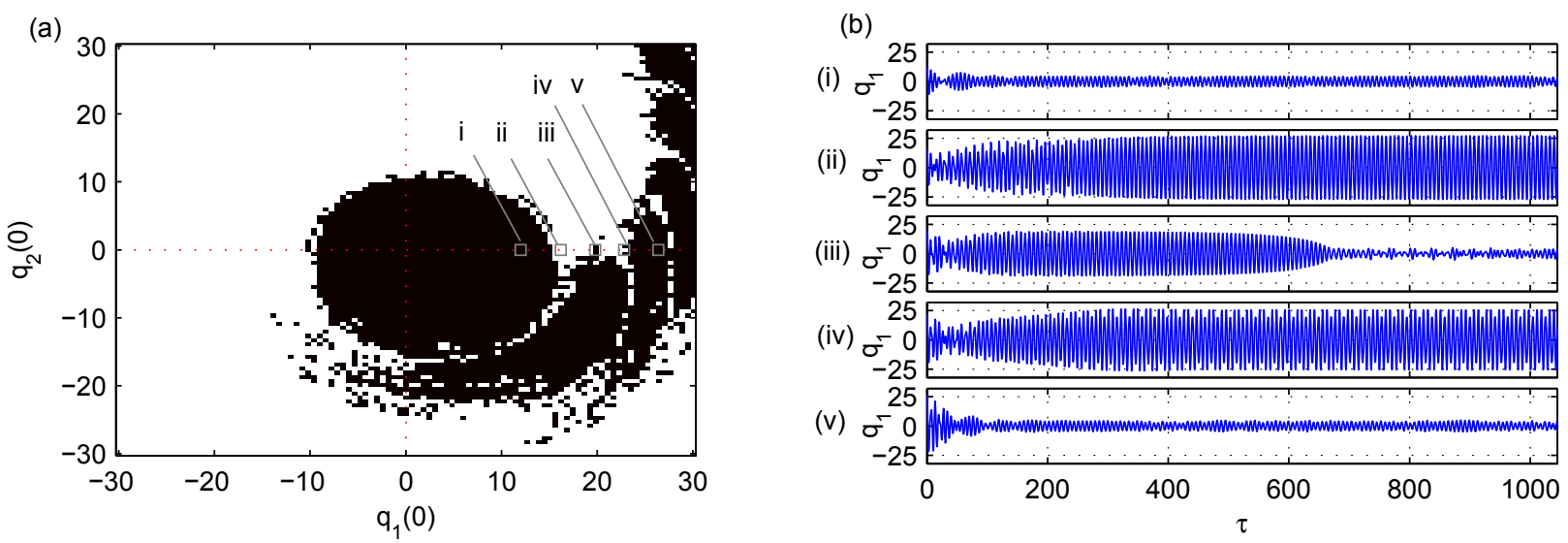

Figure 9: (a) Basin portrait and (b) corresponding $q_{1}$ time series at five points (i-v); $\Omega=0, \hat{\gamma}_{N}=0.002, \Omega_{N}=0.09, F=0.41, \phi=\pi / 2$.

of 576 basin portraits are constructed. Since each basin portrait represents the steady state behavior resulting from $101^{2}$ different initial conditions, the erosion profiles effectively summarize the behavior of the system based on a total of $5.9 \times 10^{6}$ simulations.

The erosion profiles produced through this effort are presented in Fig. 10. Each row corresponds to one of the four integrity measures versus the static displacement $F$. Zero damping and $\hat{\gamma}_{N}=0.002$ are denoted by $(\bigcirc)$ and $(+)$, and zero linear stiffness and $\Omega=0.1$ by $(-)$ and $(--)$, respectively. The integrity steadily decreases as the static displacement amplitude increases. For values of $F>0.85$ all integrity measures are approximately zero, indicating that the system will always converge to the high amplitude constraint solution.

The first conclusion regarding the erosion profiles is that the two configurations with zero damping consistently exhibit 20-40\% higher integrity than those with a small amount of damping. In addition, the zero-damping configurations maintain positive integrity for $25-45 \%$ larger static displacement values than the corresponding non-zero configurations. This indicates that when the NTMD is undamped, the primary oscillator is more likely to exhibit a low amplitude response and is more robust to impact loads. While it is impossible to truly obtain zero damping in a real-world design, it is recommended that the nonlinear absorber damping be as near-zero as possible 

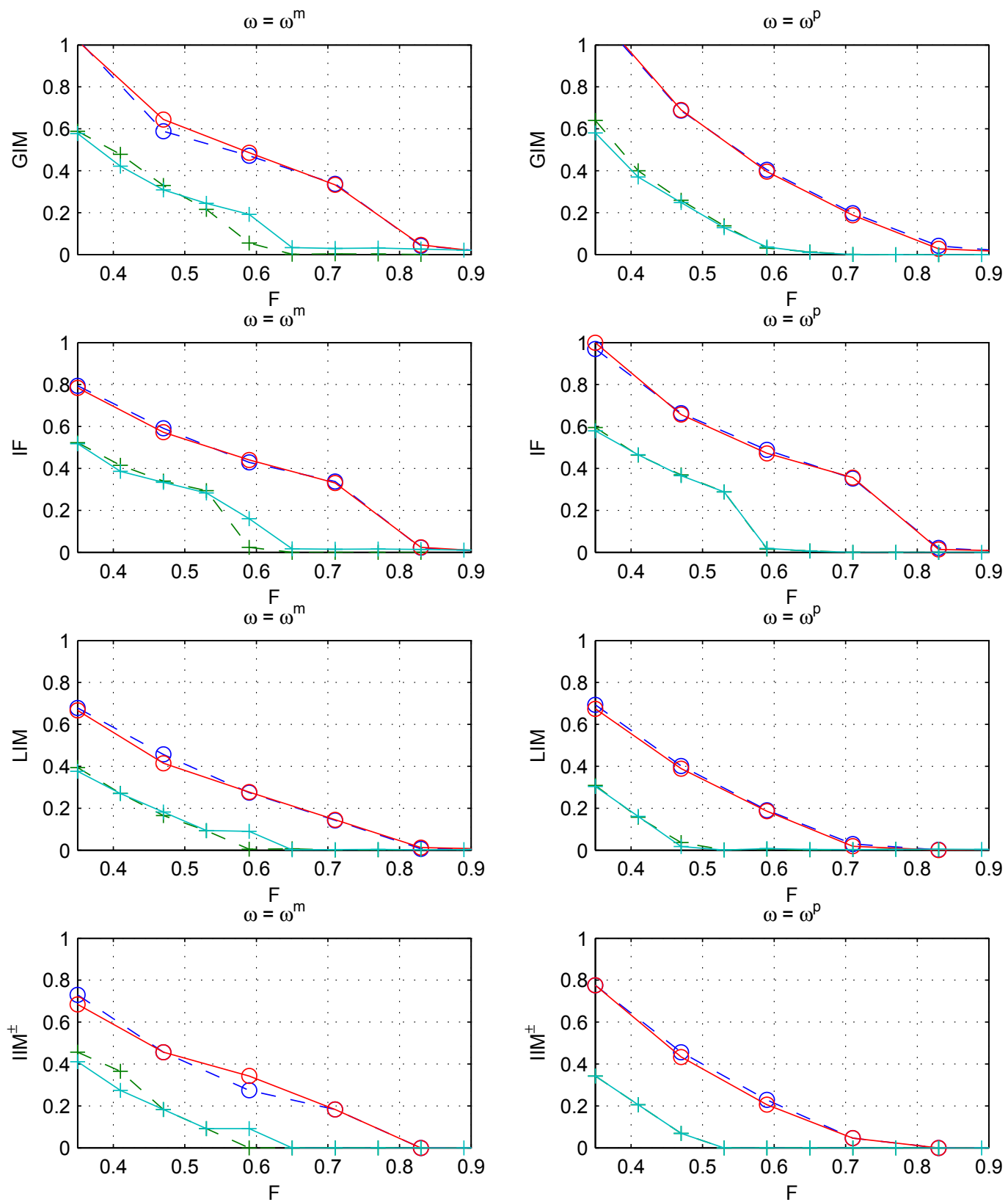

Figure 10: Erosion profiles illustrating the four integrity measures versus the static displacement at the frequencies corresponding to the midpoint (left column) and peak (right column) of $B_{\omega<1}$. 

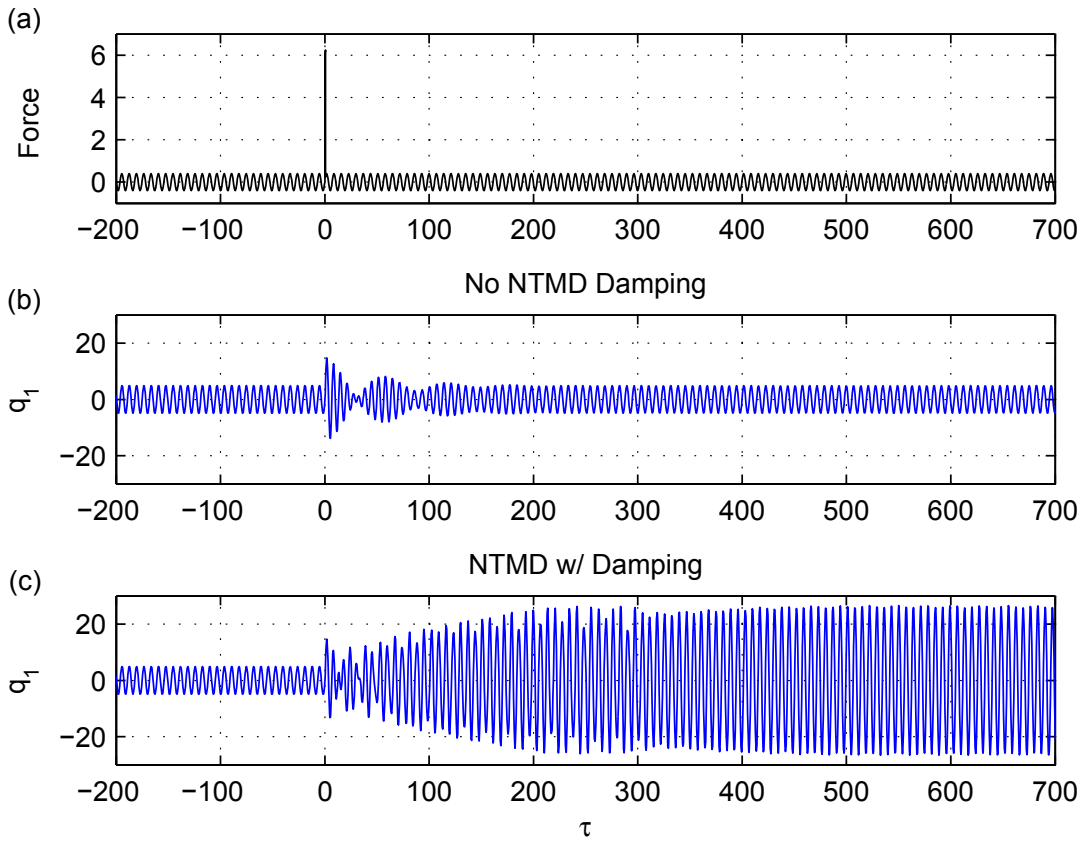

Figure 11: Time series of the (a) excitation force, (b) PS response with no NTMD damping, and (c) PS response with $\hat{\gamma}_{N}=0.002$ when the system is initially oscillating with a low amplitude and subjected to a shock load of magnitude 6.0 at $t=0 ; \Omega=0, \omega=\omega_{p}(0.896)$, $F=0.40$.

to attain the best integrity. The use of minimal damping is consistent with the design of linear TMDs, however for the NTMDs considered here this condition improves the chances of the system converging to a lower amplitude solution.

This conclusion is better illustrated by the example shown in Fig. 11. The time series of (a) excitation force, (b) PS response with no NTMD damping, and (c) PS response with $\hat{\gamma}_{N}=0.002$ is displayed. Each system is initially oscillating with a low amplitude and is then subjected to a shock load of magnitude 6.0 at $t=0$. The static displacement amplitude is $F=0.4$ and all other parameters directly correspond to the data presented in Fig. 10. It is observed in Fig. 11(b) that the system with no NTMD damping quickly recovers from the perturbation and maintains a low amplitude response, whereas the system with damping transitions to a high amplitude response as shown 
in Fig. 11(c). This behavior confirms the results presented in Fig. 10 (see the bottom right panel), which indicate that the impulse integrity, an indication of robustness to perturbations, of the system with zero damping is much higher than the system with a small amount of damping.

The second conclusion derived from the results presented in Fig. 10 is that the small linear stiffness coefficient $\Omega=0.1$ introduces no significant change to the erosion profiles, as illustrated by comparing the dashed and solid lines. Although the linear stiffness component may adversely affect other performance considerations such as transient behavior, the present study finds no evidence that a small linear stiffness component will affect the system integrity. Therefore, if a small linear stiffness component produces other favorable response characteristics, it can be added to the system without significantly impacting the integrity.

By comparing erosion profiles between the midpoint $\omega_{m}$ and peak $\omega_{p}$ of $B_{\omega<1}$ by using the GIM and IF integrity measures, we note that the integrity is consistently higher at $\omega_{p}$. This corresponds to a larger basin of attraction for the low amplitude solution when the system is excited at the higher frequency of $\omega_{p}$. As observed in Fig. $4, \omega_{p}$ is close to the excitation frequency where a cyclic-fold bifurcation of the high amplitude solution occurs and the high amplitude solution ceases to exist. It is expected that as the excitation frequency increases, the size of the constraint basin will decrease until it disappears at the bifurcation point. The larger separation between the amplitudes of the safe and constraint solutions at $\omega_{p}$ than at $\omega_{m}$ may also influence these results as more energy would be required to transition to the higher amplitude solution. However, examining the LIM and $\mathrm{IIM}^{ \pm}$ integrity measures, we observe the opposite trend that the integrity is lower $\left(\hat{\gamma}_{N}=0.002\right)$ or at best unchanged $\left(\hat{\gamma}_{N}=0\right)$ for $\omega_{p}$ when compared with $\omega_{m}$. These results indicate that although both the total hyper-volume and the size of the compact center of the safe basin increase as the frequency increases from $\omega_{m}$ to $\omega_{p}$, the trajectory of the safe attractor remains near the constraint basin boundary. Therefore, the safe solution of the system is no more robust to perturbations at the most potentially harmful frequency $\omega_{p}$ than at $\omega_{m}$. The uniform decreasing trend experienced by all four of the integrity measures reveals that the integrity of the system decreases in a consistent manner as the magnitude of the excitation increases.

Perhaps the most unexpected observation is that the safe basin integrity is completely eliminated in all four cases for $F>0.85$. By examining the various frequency-response profiles, we see that the complete loss of integrity 


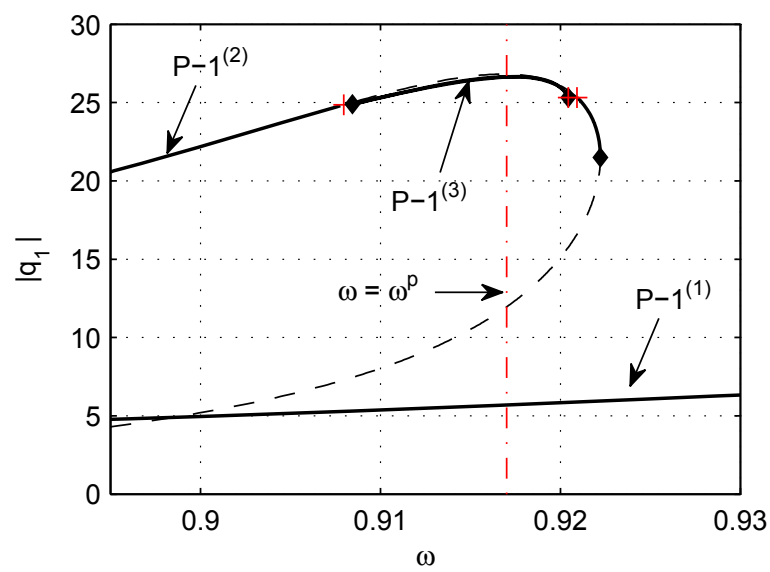

Figure 12: Appearance of a new stable Period-1 (P-1) solution which acts as a strong attractor; $\Omega=0, \hat{\gamma}_{N}=0.002, \Omega_{N}=0.09, F=0.65$. Limit point $(\diamond)$ and branch point $(+)$ bifurcation points are marked.

correlates with a bifurcation initiating at the peak of the constraint solution. These conditions are illustrated in Fig. 12 for the physically realistic case with non-zero damping where the integrity measures dropped to zero around $F=0.65$. In this bifurcation, the original constraint solution $P-1^{(2)}$ becomes unstable, but simultaneously another stable constraint solution $P-1^{(3)}$ is created. The stability change and existence of an additional stable branch in this region was also identified by [17], but the implications of this new branch on the global dynamics were not studied. Even though the original safe solution $P-1^{(1)}$ is still present and stable, the new constraint solution is a strong attractor to which all initial conditions converge. The same conditions are observed for the zero damping case when the excitation magnitude is increased to $F=0.85$.

As a final note, recall from the higher-period curves identified in Figs. 5 and 7 that the system is capable of exhibiting a Period-3 response, a characteristic typical of systems capable of exhibiting chaotic behavior. Although the intent of the present study has been fulfilled, preliminary evidence of chaotic behavior is briefly discussed. These results will be shared briefly with hopes that they may help motivate further investigations.

The distance between two trajectories initially separated by $d=1 \times 10^{-10}$ is plotted as a function of time in Fig. 13(a). Parameter values are chosen to 

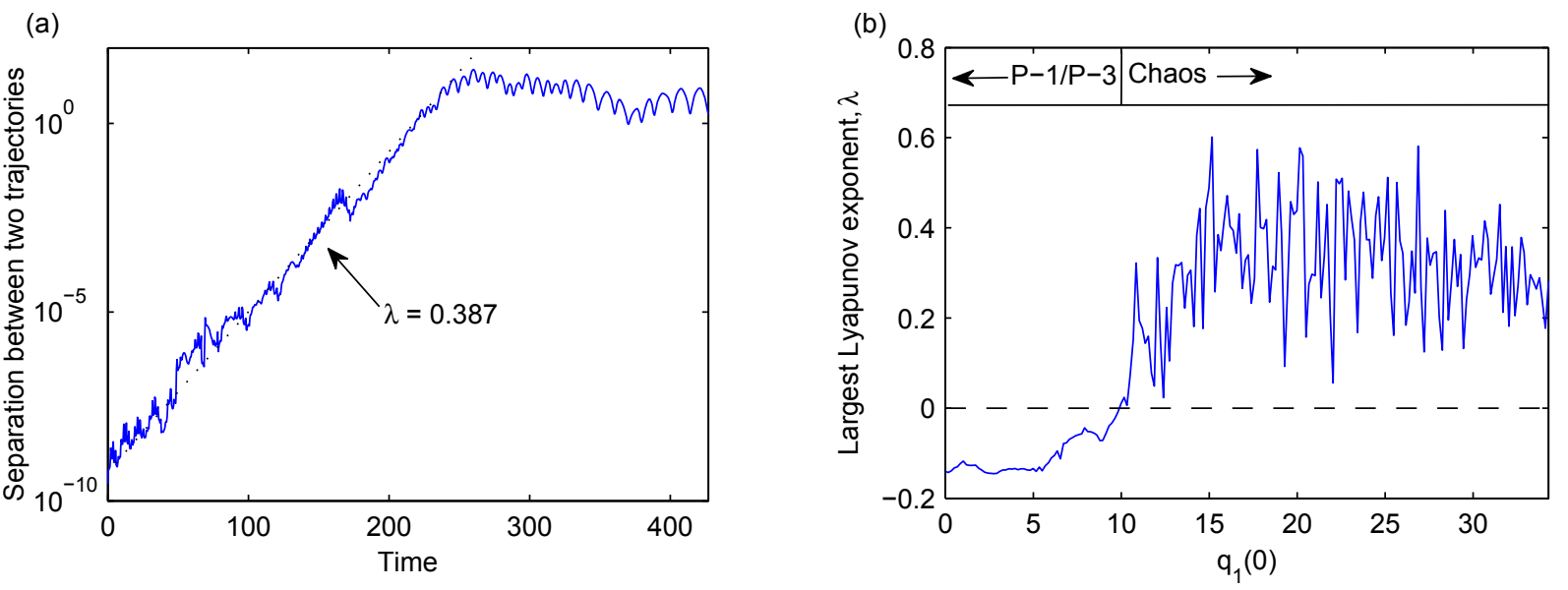

Figure 13: (a) Separation between two nearby trajectories versus time and (b) largest Lyapunov exponent versus initial position of primary oscillator, $q_{i}=0$ for $i=2,3,4$.

correspond to conditions where chaos-like behavior is observed by using $\omega=$ $1.1, \mathbf{q}^{0}=[34,0,0,0], \gamma_{N}=0.05, \Omega=0.1, \Omega_{N}=0.1$, and $F=0.35$. The plot illustrates a textbook example of exponential divergence followed by eventual saturation at $\tau \approx 250$. The largest Lyapunov exponent is positive, with a value of $\lambda=0.387$. Both are known characteristics of chaotic systems [42].

By examining the value of the largest Lyapunov exponent $\lambda$ versus the Primary initial condition $q_{1}^{0}$ in Fig. 13(b), it is observed that in the central region of the basin - for low $q_{1}^{0}$ values - the Lyapunov exponent is negative, indicating the absence of chaotic behavior. Above some threshold value however, in this case $q_{1}^{0} \geq 29, \lambda$ becomes positive. Therefore, for larger values of initial energy, it is important to consider that the present system is capable of exhibiting chaotic behavior.

\section{Concluding Remarks}

In this study, the integrity of a system consisting of a primary oscillator coupled to an absorber having a cubic stiffness nonlinearity (NTMD) was studied by using numerical methods. A series of initial simulations were used to determine the regions of parameter space within which the system behavior met two criteria: (1) multiple stable solutions coexist within the range of expected operating conditions and (2) the low amplitude response of 
the NTMD outperforms an optimally tuned linear TMD based on one or more performance measures. Based on the results from these initial simulations in addition to frequency-response curves constructed by using continuation techniques, four combinations of values for system parameters corresponding to NTMD damping and the oscillator's linear frequency ratio were selected.

Preliminary basin portraits revealed that the integrity of the safe solution within the higher-frequency bi-stable region $B_{\omega>1}$ is much higher than the integrity within the low-frequency bi-stable region $B_{\omega<1}$. Results obtained by using numerical integration confirmed that the system is much more likely to exhibit the safe, low amplitude response within $B_{\omega>1}$. This trend was especially apparent at higher frequencies, as none of the numerical integrations converged to the constraint solution at frequencies higher than the midpoint of $B_{\omega>1}$. For this reason, the in-depth study focused on $B_{\omega<1}$.

A total of 576 basin portraits were constructed by using the parallelized multi-degrees-of-freedom cell mapping method (PMDCM) to map over $5.9 \times$ $10^{6}$ response trajectories. From this data, erosion profiles were constructed, summarizing the system integrity for the four combinations of NTMD damping and the oscillator's linear frequency ratio. Based on these results, the following conclusions are made:

- Integrity values were $20-40 \%$ higher across all measures for the case of zero NTMD damping when compared with a small non-zero NTMD damping, resulting in increased robustness to large excitation magnitudes.

- A small linear stiffness coefficient $\Omega=0.1$ resulted in no significant change to the integrity measures when compared with the case of zero linear stiffness. If other favorable response characteristics can be obtained by adding or removing the small linear stiffness component, the integrity will not be affected.

- Both the total hyper-volume and the size of the compact center of the safe basin are larger at the frequency corresponding to the peak of the frequency-response $\omega_{p}$ than the midpoint of the bi-stable region $\omega_{m}$, but the distance from the attractor to the boundary of the constraint basin is generally unchanged. The corresponding local integrity measure and impulse integrity measure indicate that the system is no more robust to perturbations at $\omega_{p}$ than $\omega_{m}$, despite the larger difference in response amplitudes. 
- The integrity of the safe basin is eliminated completely at a threshold static displacement $F$ (approximately $F>0.85$ for $\hat{\gamma}_{N}=0$ and $F>0.65$ for $\hat{\gamma}_{N}=0.002$ ). The integrity loss corresponds to a bifurcation in which the original constraint solution becomes unstable and is augmented by a new stable constraint branch which acts as a strong attractor.

These results indicate that the response behavior of a strongly nonlinear absorber is more complicated than previously anticipated and that the high amplitude attractors not only exist but significantly influence the response behavior. These findings clarify the challenges that must be addressed before a strongly nonlinear absorber can be practically implemented.

Future efforts will explore the influence of noise on the integrity of these systems as well as study transient response behavior associated with broadband excitation. Experiments will also be performed in order to verify the behavior predicted by the numerical methods.

\section{References}

[1] H. Frahm, Device for damping vibrations of bodies, United States Patent No. 989,958 (1911).

[2] J. Ormondroyd, J. Den Hartog, The theory of the dynamic vibration absorber, Transactions of the ASME 50 (7) (1928) A9-A22.

[3] J. Den Hartog, Mechanical Vibrations, Dover Publishing, 1934.

[4] S. Crandall, W. Mark, Random vibration in mechanical systems, Academic Press, New York and London, 1963.

[5] P. Wirsching, G. Campbell, Minimal structural response under random excitation using the vibration absorber, Earthquake Engineering and Structural Dynamics 2 (4) (1974) 303-312.

[6] J. Brock, A note on the damped vibration absorber, Journal of Applied Mechanics 13 (4) (1946) A-284.

[7] R. Jacquot, D. Hoppe, Optimal random vibration absorbers, Journal of the Engineering Mechanics Division 99 (3) (1973) 612-616. 
[8] G. Warburton, Optimum absorber parameters for various combinations of response and excitation parameters, Earthquake Engineering and Structural Dynamics 10 (3) (1982) 381-401.

[9] R. Roberson, Synthesis of a nonlinear dynamic vibration absorber, Journal of the Franklin Institute 254 (3) (1952) 205-220.

[10] L. Pipes, Analysis of a nonlinear dynamic vibration absorber, Journal of Applied Mechanics 20 (4) (1953) 515-518.

[11] A. Nayfeh, D. Mook, Nonlinear oscillations, Wiley Interscience, New York, 1986.

[12] S. Nayfeh, A. Nayfeh, Energy transfer from high to low-frequency modes in a flexible structure via modulation, Journal of Vibration and Acoustics 116 (2) (1994) 203-207.

[13] O. Gendelman, Transition of energy to a nonlinear localized mode in a highly asymmetric system of two oscillators, Nonlinear Dynamics 25 (13) (2001) 237-253.

[14] O. Gendelman, L. Manevitch, A. Vakakis, R. M'Closkey, Energy pumping in nonlinear mechanical oscillators: Part I - dynamics of the underlying Hamiltonian systems, Journal of Applied Mechanics 68 (1) (2001) 34-41.

[15] A. Vakakis, O. Gendelman, Energy pumping in nonlinear mechanical oscillators II: Resonance capture, Journal of Applied Mechanics 68 (1) (2001) 42-48.

[16] A. Vakakis, Inducing passive nonlinear energy sinks in linear vibrating systems, Journal of Vibration and Acoustics 123 (3) (2001) 324-332.

[17] N. Alexander, F. Schilder, Exploring the performance of a nonlinear tuned mass damper, Journal of Sound and Vibration 319 (1-2) (2009) 445-462.

[18] C. Sun, R. Eason, S. Nagarajaiah, A. Dick, Hardening Duffing oscillator attenuation using a nonlinear TMD, a semi-active TMD and multiple TMD, Journal of Sound and Vibration 332 (4) (2013) 674-686. 
[19] R. Eason, C. Sun, A. Dick, S. Nagarajaiah, Attenuation of a linear oscillator using a nonlinear and a semi-active tuned mass damper in series, Journal of Sound and Vibration 332 (1) (2013) 154-166.

[20] Y. Starosvetsky, O. Gendelman, Vibration absorption in systems with a nonlinear energy sink: Nonlinear damping, Journal of Sound and Vibration 324 (3-5) (2009) 916-939.

[21] E. Doedel, AUTO: A program for the automatic bifurcation analysis of autonomous systems, Congressus Numerantium 30 (1981) 265-284.

[22] B. Tongue, On obtaining global nonlinear system characteristics through interpolated cell mapping, Physica D 28 (3) (1987) 401-408.

[23] C. Hsu, Theory of cell-to-cell mapping dynamical systems, Journal of Applied Mechanics 47 (4) (1980) 931-939.

[24] C. Hsu, R. Guttalu, Unravelling algorithm for global analysis of dynamical systems: An application of cell-to-cell mappings, Journal of Applied Mechanics 47 (4) (1980) 940-948.

[25] C. Hsu, R. Guttalu, W. Zhu, Method of analyzing generalized cell mappings, Journal of Applied Mechanics 49 (4) (1982) 885-894.

[26] B. Tongue, K. Gu, Interpolated cell mapping of dynamical systems, Journal of Applied Mechanics 55 (2) (1988) 461-466.

[27] Z.-M. Ge, S.-C. Lee, A modified interpolated cell mapping method, Journal of Sound and Vibration 199 (2) (1997) 189-206.

[28] B. Tongue, K. Gu, A higher order method of interpolated cell mapping, Journal of Sound and Vibration 125 (1) (1988) 169-179.

[29] J. van der Spek, C. de Hoon, A. de Kraker, D. van Campen, Parameter variation methods for cell mapping, Nonlinear Dynamics 7 (3) (1995) $273-284$.

[30] M. Wiercigroch, B. Kraker (Eds.), Applied nonlinear dynamics and chaos of mechanical systems with discontinuities, Vol. 28 of World Scientific Series on Nonlinear Science, World Scientific, 2000, Ch. 4, pp. 64-93. 
[31] J. van der Spek, D. van Campen, A. de Kraker, Cell mapping for multi degrees of freedom systems, in: Proceedings of the 1994 International Mechanical Engineering Congress and Exposition, Vol. 192, Chicago, IL, 1994, pp. 151-159.

[32] R. Eason, A. Dick, A parallelized multi-degrees-of-freedom cell mapping method, Nonlinear Dynamics DOI: 10.1007/s11071-014-1310-8 (2014) $1-13$.

[33] J. J. Waite, L. N. Virgin, R. Wiebe, Competing responses in a discrete mechanical system, International Journal of Bifurcation and Chaos 24 (2014) 1-13, art. no. 1430003.

[34] Y. Ueda, M. Hirano, H. Ohta, R. H. Abraham, Attractor and basin portraints of a double swing power system, International Journal of Bifurcation and Chaos 14 (9) (2004) 3135-3152.

[35] G. Rega, S. Lenci, Dynamical integrity and control of nonlinear mechanical oscillators, Journal of Vibration and Control 14 (1-2) (2008) 159-179.

[36] D. D. Quinn, A. L. Triplett, L. A. Bergman, A. F. Vakakis, Comparing linear and essentially nonlinear vibration-based energy harvesting, Journal of Vibration and Acoustics 133 (2011) 1-8, art. no. 011001.

[37] L. Ruzziconi, M. I. Younis, S. Lenci, Multistability in an electrically actuated carbon nanotube: A dynamical integrity perspective, Nonlinear Dynamics 74 (3) (2013) 533-549.

[38] G. Rega, V. Settimi, Bifurcation, response scenarios and dynamic integrity in a single-mode model of noncontact atomic force microscopy, Nonlinear Dynamics 73 (1-2) (2013) 101-123.

[39] S. Lenci, G. Rega, L. Ruzziconi, The dynamical integrity concept for interpreting/predicting experimental behaviour: From macro- to nanomechanics, Philosophical Transactions of the Royal Society A 371 (2013) $1-19$.

[40] M. Soliman, J. Thompson, Integrity measures quantifying the erosion of smooth and fractal basins of attraction, Journal of Sound and Vibration 135 (3) (1989) 453-475. 
[41] S. Lenci, G. Rega, Optimal control of nonregular dynamics in a Duffing oscillator, Nonlinear Dynamics 33 (1) (2003) 71-86.

[42] A. Nayfeh, B. Balachandran, Applied nonlinear dynamics, Wiley-VCH, Weinheim, 2004. 